\title{
Mathematical Modeling as an Accurate Predictive Tool in Capillary and Microstructured Fiber Manufacture: The Effects of Preform Rotation
}

\author{
Christopher J. Voyce, Alistair D. Fitt, and Tanya M. Monro
}

\begin{abstract}
A method for modeling the fabrication of capillary tubes is developed that includes the effects of preform rotation, and is used to reduce or remove polarization mode dispersion and fiber birefringence. The model is solved numerically, making use of extensive experimental investigations into furnace temperature profiles and silica glass viscosities, without the use of fitting parameters. Accurate predictions of the geometry of spun capillary tubes are made and compared directly with experimental results, showing remarkable agreement and demonstrating that the mathematical modeling of fiber drawing promises to be an accurate predictive tool for experimenters. Finally, a discussion of how this model impacts on the rotation of more general microstructured optical fiber preforms is given.
\end{abstract}

Index Terms-Mathematical modeling, optical fiber, optical fiber applications, optical fiber fabrication, optical fiber theory.

\section{INTRODUCTION}

I N A microstructured optical fiber (MOF), an arrangement of air holes surrounds the fiber core (which can be made of glass or air). The use of air holes to form the fiber cladding region means that these fibers can be made from a single piece of glass. When a solid core is used, the presence of air holes lowers the effective refractive-index, and the effective refractive-index difference allows the fiber to guide light [1]-[3]. Although at first sight this guidance mechanism is similar to that operating in a conventional optical fiber, microstructured fibers exhibit a host of highly unusual and tailorable optical properties. They may be manufactured by heating a macroscopic structured preform (typically a few centimeters in diameter), and drawing it down to the required dimensions (typically $125 \mu \mathrm{m}$ in diameter). The presence of air holes in the cross-section presents both challenges and opportunities for the fabrication of these fibers. Competition between viscosity and surface tension effects can alter the size and shape of the holes during the drawing process, and in extreme cases, hole closure may even occur. Since the fabrication of structured preforms is one of the most labor intensive parts of the manufacturing process, it is often desirable

Manuscript received August 6, 2007; revised December 9, 2007.

C. J. Voyce is with the Mathematical Institute, University of Oxford, Oxford OX1 3LB, U.K. (e-mail: voyce@maths.ox.ac.uk).

A. D. Fitt is with the School of Mathematics, University of Southampton, Southampton SO17 1BJ, U.K. (e-mail: adf@maths.soton.ac.uk).

T. M. Monro is with the Centre of Expertise in Photonics, University of Adelaide, Adelaide SA 5005, Australia (e-mail: tanya.monro@adelaide.edu.au).

Color versions of one or more of the figures in this paper are available online at http://ieeexplore.ieee.org.

Digital Object Identifier 10.1109/JLT.2007.914515 to produce a number of different fiber profiles from a single preform by altering the conditions under which the fiber is drawn. Predictive modeling capabilities are therefore crucial, together with the ability to understand and control undesirable effects such as hole shape change and hole closure.

Conventional (solid) preforms are routinely rotated to introduce a twist into the final fiber, since this reduces polarization mode dispersion (PMD) [4]-[6], an effect that is often regarded as undesirable. The periodicity of the twist required depends on the wavelength of light and the details of the fiber profile. Fiber preforms are normally rotated at the furnace inlet and held with zero rotation at the outlet, leaving the fiber with an overall twist along its length [7]. This strategy succeeds provided the twist periodicity (spin pitch) does not exceed the beat length, typically in the range $0.1 \mathrm{~mm}-10 \mathrm{~cm}$. However, there is currently a practical limit of about $2000 \mathrm{r} / \mathrm{min}$ to the rate at which preforms may be rotated. At rates greater than this, the alignment of the preform in the furnace becomes unstable and the preform begins to vibrate [8].

Preservation of the microstructure has recently been demonstrated when rotating large mode-area fibers with a high density of small, dispersed holes, at rates sufficient to dramatically reduce PMD [7]. However, as discussed in [9], rotation can sometimes affect fiber geometry, thus suggesting the possibility of using preform rotation as an additional control parameter in the drawing process.

\section{INTRODUCTION TO FLOW MODELING}

Evidently microstructured-fiber technology would be greatly aided by the development of accurate tools for predicting the changes that occur during fiber drawing. In a previous study, we developed a mathematical model to examine the effects of preform rotation on capillary drawing [9]. While it may appear that a capillary tube is an unrealistic approximation of a complex microstructured fiber, for the two specific fiber-types described in [10] ("Type-one" fibers: large mode-area microstructured fibers with an approximately uniform distribution of diffuse holes in a cross section, and "Type-two fibers": fibers where a large jacket surrounds a microstructured region with a high density of holes in a cross section), the approximation is justified and thus the results of this paper may be directly applied to microstructured fiber preform spinning for certain classes of microstructured fiber structure (see [10] for details). Furthermore, we regard the model described here and detailed in [9] as an intermediate step towards modeling the fabrication of arbitrary MOFs. 
There exist a number of studies of optical-fiber drawing, including studies related to the drawing of capillary tubes (see, for example, [9]-[14]). However, the desire to reduce fiber birefringence and PMD in microstructured fibers motivates an analysis that additionally includes the effects of preform rotation on drawn fibers.

The practical usefulness of mathematical modeling of this type can take several forms: 1) the identification of useful parameters (e.g., sensitivity parameters to determine stable drawing regimes; see [12]); 2) an increased understanding of the physics involved in the problem (see [10] and [13] for a predominantly qualitative discussion of the effects of preform rotation); and 3) accurate predictions of the appropriate choices of drawing parameters for given geometric requirements.

The ability of mathematical modeling to achieve 3) is limited by the accuracy with which the experimental variables are known. For example, glass viscosity varies markedly with temperature, is often proprietary information, and is particularly sensitive to the fraction of water and other impurities present in the glass (especially in the case of silica, the glass traditionally used in microstructured fiber manufacture). Also, temperature gradients in the furnace often depend on the flow of gas within the furnace.

The development of complete mathematical models that explain some of the undesirable features such as hole distortion observed in MOF drawing is some way off, and to date, the verification of mathematical models for solid optical fiber drawing and capillary tube drawing has been limited by the presence of fitting parameters specifically chosen to achieve good agreement between theoretical predictions and experimental results, decreasing confidence in the absolute predictive power of the models. For example, [11] successfully compared the results of theoretical predictions for inner and outer diameter with experimental results, but because it can be difficult to ascertain the absolute glass temperature during fiber drawing, the authors state that "while the three drawing temperatures are likely to be accurate relative to each other, it is possible that they may suffer from an absolute temperature offset by as much as $50^{\circ} \mathrm{C}$..., we therefore fitted the theoretical model to the experimental data by choosing the parameter $\gamma / \mu$ [surface tension to viscosity ratio]".

\section{AIMS}

Further to the results of [9] and [10], the aims of this study are therefore to 1) confirm that preform rotation causes geometry change, to predict these changes and compare the predictions with experimental results and 2) to validate the mathematical model (described below) by carefully establishing all experimental variables, thus removing the need for fitting parameters. It is hoped that this will both increase the confidence held in mathematical modeling, promoting its continued development for complex MOF manufacture, and provide an accurate predictive tool to experimenters for certain classes of MOF (see [9] for a detailed discussion of how the mathematical model may be used to predict geometry changes in general classes of MOF).

This paper is therefore unique in its integration of mathematical modeling, extensive experimental research, and numerical analysis; providing experimenters with the first "black box" tool for optical fiber drawing.
The remainder of this paper is organized as follows. In Section IV, we give details of experiments conducted to establish the furnace temperature profiles at a variety of temperatures and discuss the appropriate viscosity law to implement. In Section V, we present a brief exposition of the mathematical model reported in [9], which we solve numerically using a Fortran-77 code that employs standard Numerical Algorithms Group Ltd. (NAG) routines. In Section VI, we detail the experimental results and numerical predictions of the model for the drawing of a capillary tube at a variety of furnace temperatures and preform rotation rates. We discuss the findings in Section VII and summarize the main results of this study in Section VIII.

\section{EXPERIMENTAL STUDY}

\section{A. Motivation}

Mathematical models for optical fiber drawing require input data such as the feed speed, draw speed, and preform geometry, all of which may be measured to a high degree of accuracy. Models also require specification of the viscosity of the glass as a function of position. This is equivalent to requiring the specification of the furnace temperature profile and the temperature dependence of the glass viscosity, which is a strong function of temperature at typical drawing temperatures.

A numerical study of the equations that govern fiber drawing [(1)-(4) of Section V; see [14] for details] reveals that when preform rotation affects fiber geometry, the geometry is sensitive to small changes in the viscosity and the viscosity gradient. In order to make comparisons with experimental data while avoiding the need to use the viscosity, surface tension or a combination of the two as fitting parameters [11] it is necessary to accurately determine both the temperature dependence of the glass viscosity and the temperature distribution within the furnace.

\section{B. Furnace Temperature Profiles}

In practice, furnace temperature profiles vary between drawing towers and furnace designs, as well as depending on the particular setup of the furnace (susceptors and gas flows may be varied). Furthermore, furnace temperature profiles change with time as the heating elements degrade.

Further complications arise from the fact that the length of the furnace is difficult to estimate, since the effective heating-region depends on the temperature of the furnace. The furnace length in the models represents the length of preform considered fluid (i.e., the length of preform whose shape can change through stretching, etc.). Because the furnace is not isothermal and often has a temperature maximum near the midpoint of the furnace, the effective furnace length increases with increasing temperature.

For the reasons given, we proceed to describe our choice of viscosity law and the experiments performed to determine the temperature profile of the drawing furnace on which the spinning experiments in this paper were conducted. (Note that the information on temperature profiles presented below is specific to the drawing tower used in our experiments and is not likely to accurately represent drawing towers in general, though the 
process of obtaining such information is crucial to verify the mathematical modeling concepts and develop them as an accurate predictive tool for experimenters.)

\section{Viscosity of Silica Glass}

The glass used in the experiments conducted was undoped Suprasil F300, a silica glass commonly used in the production of high-quality MOFs. The viscosity of Suprasil F300 and its temperature dependence is proprietary information and accurate data is therefore not publicly available. The precise value of the surface tension of the glass is also unknown, as is its temperature variation.

Previous mathematical studies have assumed that the drawing furnace has a constant temperature whereas in reality the temperature is expected to vary significantly over the length of the furnace. While obtaining accurate data to remove all unknowns is unrealistic, a concerted effort was made to reduce the uncertainties. Doremus [15] was contacted and upon his advice, it was decided that the viscosity measurements carried out by Hetherington et al. [16] most accurately represent the viscosity of Suprasil F300 glass for $1000{ }^{\circ} \mathrm{C} \leq T \leq 1400{ }^{\circ} \mathrm{C}$, and for $1400{ }^{\circ} \mathrm{C} \leq T \leq 2500{ }^{\circ} \mathrm{C}$ those of Urbain et al. [17] should be used. It should be noted that the concentration of water and other impurities can dramatically affect the viscosity curve for silica glasses and that great care must therefore be taken when choosing an appropriate viscosity curve. Furthermore, the standard methods used to measure viscosity experimentally have varying degrees of accuracy, depending on the temperature range of interest [15]. The experimentally measured viscosity law used in our numerical simulations is given in [17] as

$$
\mu=5.8 \times 10^{-7} \exp \left(\frac{515400}{(8.3145 T+2271.10567)}\right) \text { Poise }
$$

For details on how viscosity was measured, how the techniques vary with temperature, and a more general discussion of these results, see [15].

\section{Determination of the Furnace Temperature Profile}

In order to determine the temperature profile of the drawing furnace, a thermocouple (accurate to $0.4{ }^{\circ} \mathrm{C}$ ) was inserted into the air-hole in a capillary tube $10 \mathrm{~mm}$ in diameter (similar to the size of capillary tube used to manufacture microstructured fibers). The preform containing the thermocouple was passed into the furnace and fed slowly at $5 \mathrm{~mm} / \mathrm{min}$ along the furnace length to ensure thermal equilibrium at all times. The specified peak temperature (the temperature as set by the furnace control mechanism, which is measured by a pyrometer located next to a furnace element) was set to $1300^{\circ} \mathrm{C}, 1600{ }^{\circ} \mathrm{C}$, and $1760^{\circ} \mathrm{C}$, respectively, with measurements of thermocouple temperature being taken at each separate setting. The temperature of the furnace is then assumed equal to the temperature of the preform. In any case, it is the temperature of the preform that determines the fiber geometry through the glass viscosity.

Two pieces of information are required to solve the mathematical model numerically. First, the shape of the furnace temperature profile and knowledge of whether or not this shape de-

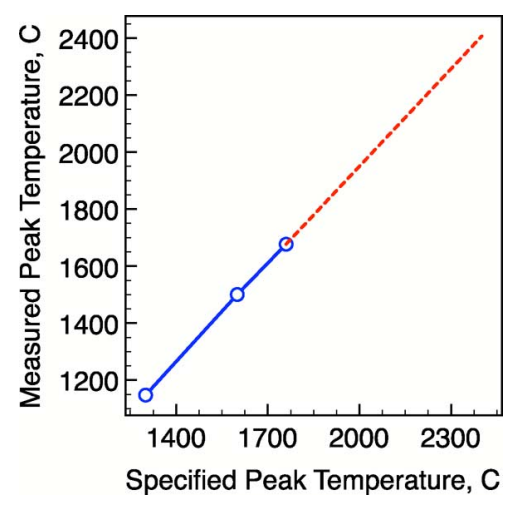

Fig. 1. Comparison between specified and measured peak temperatures of the furnace. (Symbols: Measured values. Dashed lines: Projected values.)

pends on peak temperature is required. Second, the difference between the specified peak temperature of the furnace and the measured peak temperature is required. If it is established that the temperature profile shape is independent of peak temperature, then the "real" temperature profile may be obtained from a knowledge of the shape of the furnace temperature profile and the relationship between the specified and measured peak temperatures, removing the need to measure the temperature profile at all peak temperatures of interest.

The results of furnace profile measurements are presented in Fig. 1, which shows a plot of the specified peak temperature versus measured peak temperature. Since the temperature cannot be measured at operating temperatures (the thermocouple becomes inaccurate at $1700^{\circ} \mathrm{C}$ before melting at higher temperatures), furnace temperature measurements were made at lower temperatures and the comparisons between the measured values of peak temperature and the specified peak temperature were extrapolated to the temperature range of interest $\left(2000^{\circ} \mathrm{C}-2200^{\circ} \mathrm{C}\right.$ ) (dashed line in Fig. 1). Fig. 2 shows a comparison of the profile shapes at the different peak temperatures, where the data has been shifted so that the peak temperatures coincide. It shows clearly that the assumption of a constant profile shape is acceptable, given that for the purposes of the mathematical modeling, the length of the furnace is approximately $30 \mathrm{~mm}$. The difference in the position of the peaks should be expected, since as the furnace temperature changes, the relative importance of the heat transfer processes will also change.

While in the temperature range of interest the specified peak temperature is lower than the actual peak temperature, the implication of Fig. 1 is that at large enough temperatures the specified peak temperature will in fact be higher than the measured peak temperature. Any error in this assumption is likely to be small over such small temperature ranges as those considered (approximately $200^{\circ} \mathrm{C}$ ).

\section{MODELING DESCRIPTION}

A schematic diagram of the capillary geometry is shown in Fig. 3 and a full exposition of the mathematical modeling is contained in [9]. To develop a mathematical model for the process of capillary drawing that is capable of including the effects of internal hole pressurization, surface tension, gravity and inertia as well as the effects of preform rotation, we begin 

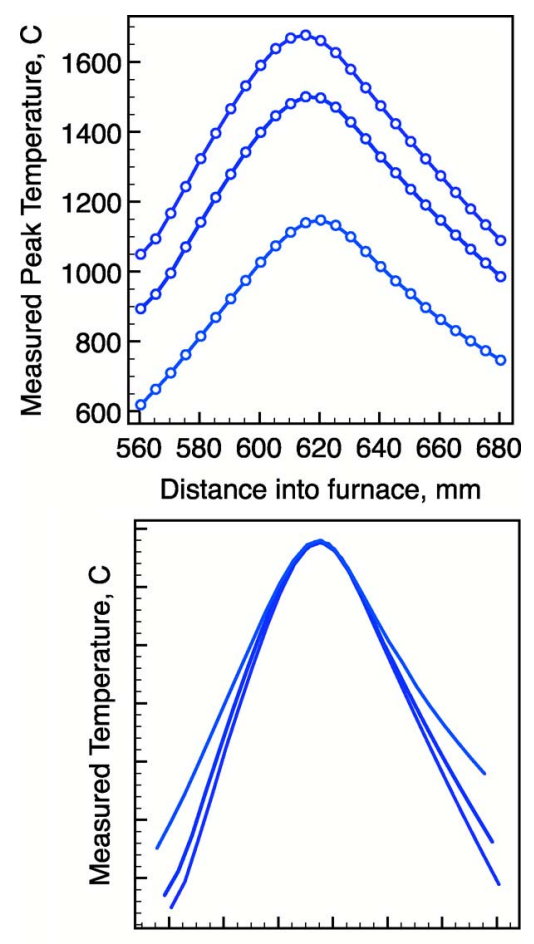

Distance into furnace, $\mathrm{mm}$

Fig. 2. Graphs to allow the comparison of all three temperature profiles. (Upper diagram: Actual values. Lower diagram: Values shifted to allow comparison of shape.)

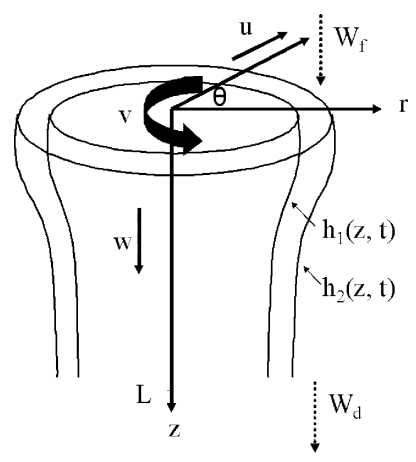

Fig. 3. Problem geometry.

with the Navier-Stokes equations using cylindrical coordinates. The glass viscosity is assumed to be a function of both axial and radial position.

We non-dimensionalize the governing equations, allowing us to simplify the equations by taking advantage of the slender preform geometry. Extensive asymptotic analysis allows the equations to be solved in closed form in certain limiting cases (e.g., ignoring the effects of gravity, inertia, and surface tension). We apply the same simplifying approximations to the physical boundary conditions, before finally deriving a closed system of "leading-order" equations that describe the drawing of rotating preforms. The free parameters in the model include: 1) glass viscosity (determined by the glass properties and the furnace temperature profile); 2) surface tension; 3 ) the length of the drawing furnace; 4 ) the initial preform geometry (i.e., inner and outer diameters); and 5) the feeding and drawing speeds.
TABLE I

NOTATION

\begin{tabular}{ll}
\hline Symbol & Definition \\
\hline & \\
\hline & time \\
$r$ & distance along capillary axis \\
$p_{0}$ & distance perpendicular to axis \\
$g$ & hole overpressure \\
$\rho$ & acceleration due to gravity \\
$\mu$ & density \\
$\gamma$ & dynamic viscosity \\
$L$ & surface tension \\
$T$ & furnace length \\
$B$ & temperature \\
$B_{0}$ & angular frequency \\
$w_{0}$ & preform angular frequency \\
$W_{f}$ & feeding-order downstream fluid velocity \\
$W_{d}$ & drawing speed for freform \\
$h_{2}$ & outer radius \\
$h_{1}$ & inner radius \\
$h_{20}$ & initial outer preform radius \\
$h_{10}$ & initial inner preform radius
\end{tabular}

The final equations are given by

$$
\begin{aligned}
& \rho\left(h_{2}^{2}-h_{1}^{2}\right)\left(w_{0 t}+w_{0} w_{0 z}-g\right) \\
& =\left[3 \mu\left(h_{2}^{2}-h_{1}^{2}\right) w_{0 z}+\gamma\left(h_{1}+h_{2}\right)+\frac{\rho}{4}\left(h_{2}^{4}-h_{1}^{4}\right) B^{2}\right]_{z} \\
& \left(h_{1}^{2}\right)_{t}+\left(h_{1}^{2} w_{0}\right)_{z} \\
& =\frac{h_{1} h_{2}\left[2 p_{0} h_{1} h_{2}-2 \gamma\left(h_{1}+h_{2}\right)+\rho h_{1} h_{2} B^{2}\left(h_{2}^{2}-h_{1}^{2}\right)\right]}{2 \mu\left(h_{2}^{2}-h_{1}^{2}\right)} \\
& \left(h_{2}^{2}\right)_{t}+\left(h_{2}^{2} w_{0}\right)_{z} \\
& =\frac{h_{1} h_{2}\left[2 p_{0} h_{1} h_{2}-2 \gamma\left(h_{1}+h_{2}\right)+\rho h_{1} h_{2} B^{2}\left(h_{2}^{2}-h_{1}^{2}\right)\right]}{2 \mu\left(h_{2}^{2}-h_{1}^{2}\right)} \\
& \rho\left[h_{2}^{2}\left(h_{2}^{2} B\right)_{t}-h_{1}^{2}\left(h_{1}^{2} B\right)_{t}\right]+\rho w_{0}\left[h_{2}^{2}\left(h_{2}^{2} B\right)_{z}-h_{1}^{2}\left(h_{1}^{2} B\right)_{z}\right] \\
& \quad+\frac{\rho}{\mu} p_{0} B h_{1}^{2} h_{2}^{2}-\frac{\rho \gamma B h_{1} h_{2}}{\mu}\left(h_{1}+h_{2}\right) \\
& \quad+\frac{\rho^{2} B^{3} h_{1}^{2} h_{2}^{2}}{2 \mu}\left(h_{2}^{2}-h_{1}^{2}\right)=\mu\left[\left(h_{2}^{4}-h_{1}^{4}\right) B_{z}\right]_{z}
\end{aligned}
$$

and the boundary conditions are given by

$$
\begin{aligned}
h_{1}(0) & =h_{10}, \quad h_{2}(0)=h_{20}, \quad w_{0}(0)=W_{f}, \\
w_{0}(L) & =W_{d}, \quad B(0)=B_{0}, \quad B(L)=0
\end{aligned}
$$

where all notation is defined in Table I. Subscripts denote differentiation.

Equations (1)-(5) are a system of partial differential equations with a two-point boundary value problem that must be solved numerically. We consider only the steady-state case and employ standard library routines by implementing the NAG routine D02HAF [18], which uses an efficient Runge-KuttaMerson method. It is not our intention here to discuss (1)-(5), and we instead refer the reader to Voyce et al. [9]. 


\section{EXPERIMENTAL FIBER DRAWS AND COMPARISON WITH NUMERICAL RESULTS}

We now present the result of detailed numerical/experimental comparisons for capillary tubes. The density of the glass is known to be $2200 \mathrm{~kg} / \mathrm{m}^{3}$ and the acceleration due to gravity was taken to be $9.81 \mathrm{~m} / \mathrm{s}^{2}$, leaving only the surface tension unspecified. To our best estimate the surface tension does not vary significantly with temperature, and is likely to be about $0.3 \mathrm{~N} / \mathrm{m}$ [12].

Since the shape of the temperature profile does not depend critically on the furnace temperature we shall assume a given furnace profile and simply adjust the peak temperature of the profile. Furthermore, we shall convert the specified peak temperature into measured peak temperatures in order to ensure the correct glass viscosity is used using Fig. 1.

The peak temperature was assumed to correspond to the temperature measured at the mid-point of the furnace, i.e., $z=L / 2$. Ideally, one should include the whole temperature profile of the furnace and run simulations to obtain the final fiber geometries. However, the temperature in the upper and lower portions of the furnace is below the softening point of the glass (approximately $1600{ }^{\circ} \mathrm{C}$ ). In these regions, the two fiber radii and the downstream velocity are constant and all derivatives are zero. If the totality of glass in the furnace were to be modeled, solving the problem by shooting methods would be problematic. Therefore, only the portion of the furnace that contains glass at a temperature above the softening point is included in the numerical model. This is achieved numerically by setting the draw length $L$ to be $2 \mathrm{~cm}$ (a deliberate underestimate), before gradually increasing the value for $L$ until the resulting fiber geometry remains constant on further increases of $L$. The value of $L$ for which this occurs is then used for all numerical/experimental comparisons.

Results are presented for the drawing of a capillary tube with an initial outer diameter (OD) of $9.95 \mathrm{~mm}$ and inner diameter (ID) of $2.85 \mathrm{~mm}$. The other experimental parameters were $W_{f}=8.83 \times 10^{-5}, W_{d}=1.00 \times 10^{-1}$. The value of parameters used to make numerical predictions were $h_{10}=1.425 \times 10^{-3}$, $h_{20}=4.975 \times 10^{-3}, W_{f}=8.83 \times 10^{-5}, W_{d}=1.0 \times 10^{-1}$, $\gamma=3 \times 10^{-1}, L=3 \times 10^{-2}$, and $g=9.81$, where all units are assumed S.I. unless otherwise stated. The same capillary tube was used for all experimental draws, which were conducted on the same day to ensure consistent alignment of the capillary tube in the furnace.

The capillary tube was drawn using the experimental parameters listed above at peak furnace temperatures of $2100{ }^{\circ} \mathrm{C}$, $2150^{\circ} \mathrm{C}$, and $2200^{\circ} \mathrm{C}$. At each temperature, the rotation rate of the capillary tube was incrementally increased from zero to the maximum value possible before mechanical oscillations of the capillary tube in the furnace were observed. Fiber samples were taken for each rotation rate at each temperature after a steady state had been reached, and the inner and outer diameters were measured using an optical microscope.

Fig. 4 shows the comparison between predicted and experimentally measured inner fiber diameters for varying rotation rates, at each of the three temperatures considered. The reason for not showing the results of measurements for the outer diameter is that even for the largest rotation rates used in these
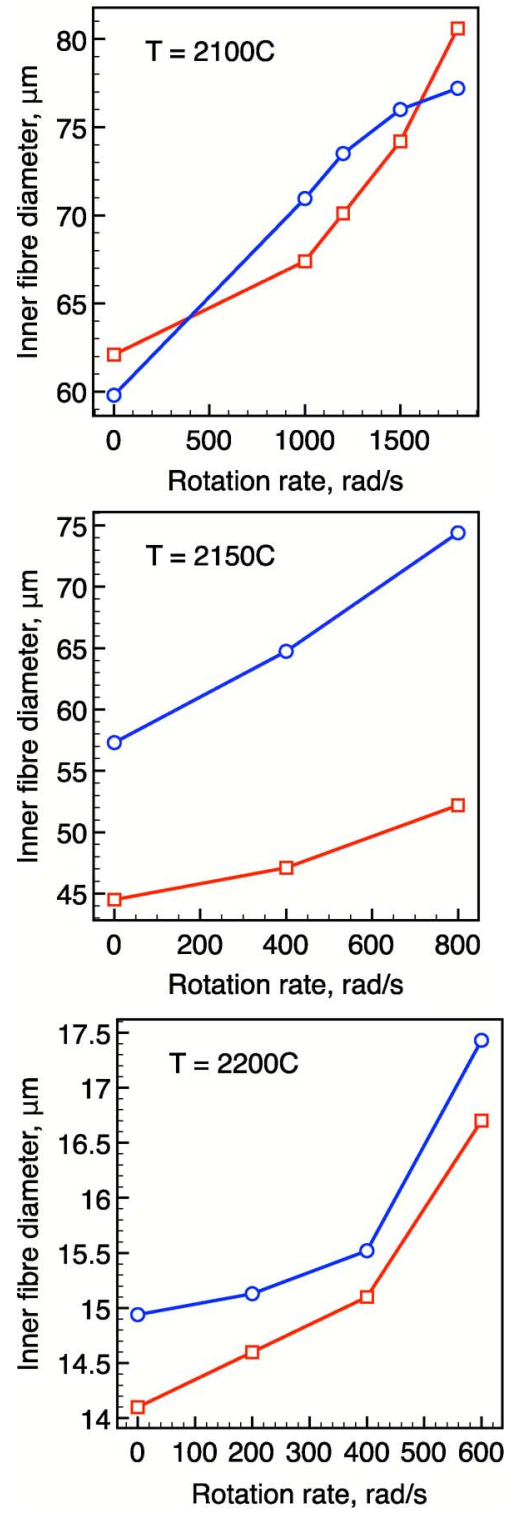

Fig. 4. Graphs showing comparison between predicted (squares) and experimentally observed (circles) inner fiber diameters at various capillary tube rotation rates for $\mathrm{T}=2100{ }^{\circ} \mathrm{C}$ (top), $\mathrm{T}=2150{ }^{\circ} \mathrm{C}$ (center) and $\mathrm{T}=2200^{\circ} \mathrm{C}$ (bottom). (Experimental parameters: $h_{10}=1.425 \times 10^{-3}, h_{20}=4.975 \times$ $10^{-3}, W_{f}=8.83 \times 10^{-5}, W_{d}=1.00 \times 10^{-1}$. Modeling parameters: $h_{10}=$ $1.425 \times 10^{-3}, h_{20}=4.975 \times 10^{-3}, W_{f}=8.83 \times 10^{-5}, W_{d}=1.0 \times 10^{-1}$, $T=2050, \gamma=3 \times 10^{-1}, L=3 \times 10^{-2}, g=9.81$. S.I. units are assumed.)

experiments, the change in size of the outer diameter (size with rotation compared to size with no rotation) was $\pm 1 \mu$, which is similar to the error in the measurement of the outer diameter. For a detailed discussion of why preform rotation does not significantly affect the outer diameter, together with numerical examples, see [10] and [13].

\section{DISCUSSION}

The first point to note about the results in Fig. 4 is that fiber geometry is significantly affected by changes in the rotation rate, as predicted by the mathematical modeling. Thus, rotation does indeed provide an additional experimental input parameter that may be used to control the geometry of MOFs. 
The results in Fig. 4 show that the error in predicted values varies between approximately $3 \%$ and $21 \%$, which we consider to represent excellent agreement given the uncertainty and possible error in the viscosity assumed, the value for surface tension used, and the possible error in the furnace temperature profile. Such good agreement betters the previous agreement of approximately $20 \%$ error achieved in [11] with the use of a fitting parameter.

There is no clear trend of agreement increasing or decreasing between experiments conducted at different temperatures as might be expected if the error were systematic. For example, if the viscosity law used was incorrect, one might expect the difference between predicted and experimental values to increase with increasing temperature, since the viscosity depends exponentially on temperature.

The trends predicted numerically for different furnace temperatures all suggest that the rate of increase of inner diameter with temperature increases with increasing rotation rate. This trend is observed experimentally for $\mathrm{T}=2150{ }^{\circ} \mathrm{C}$ and $\mathrm{T}=$ $2200{ }^{\circ} \mathrm{C}$, but not for $\mathrm{T}=2100^{\circ} \mathrm{C}$. We suggest that this results from an error in the experimentally observed value of inner diameter for $\mathrm{T}=2100$ and a rotation rate of $1800 \mathrm{rad} / \mathrm{s}$. Thus, the fiber geometry becomes more sensitive to changes in rotation rate at large rotation rates. This has possible implications for the manufacture of fibers with small holes. The drawing of fibers with small holes is problematic since the effects of surface tension are significant, and holes are therefore particularly sensitive to the experimental setup and input parameters, often leading to the closure of holes. Both pressure control [11] and the control of hole size by preform rotation cause greater changes of hole size with increasing pressure and rotation rate, respectively. However, we suggest that using both mechanisms simultaneously may remove this problem, for example by pressurizing a capillary tube and then rotating it, then requiring only small rotation rates to fine-tune the hole size.

\section{A. Air-Filling Fraction}

The air-filling fraction (defined as $h_{1} / h_{2}$ ) is an important quantity in MOF manufacture since it characterizes the optical properties of a MOF such as mode area, nonlinearity, confinement loss, and the number of guided modes the fiber can support.

Fig. 5 shows good agreement between experimental and predicted air-filling fractions and demonstrates a clear trend for the air-filling fraction to increase as the preform rotation rate is increased.

The reason that the air-filling fraction increases with increasing rotation rates is as follows. As the preform is rotated the radii of both the inner and the outer wall increase. Numerical calculations confirm that the difference between the expansion of the inner and outer walls increases as the rotation rate increases. It is trivial to show that preform rotation leads to (a) the air-filling fraction with rotation to be larger than the air-filling fraction without rotation, and further possible to show that rotation leads to (b) an air-filling fraction that increases monotonically with increasing $B_{0}$ (increasing preform rotation rate) for all rotation rates and for all values of experimental input parameters. This means that the air-filling fraction be-
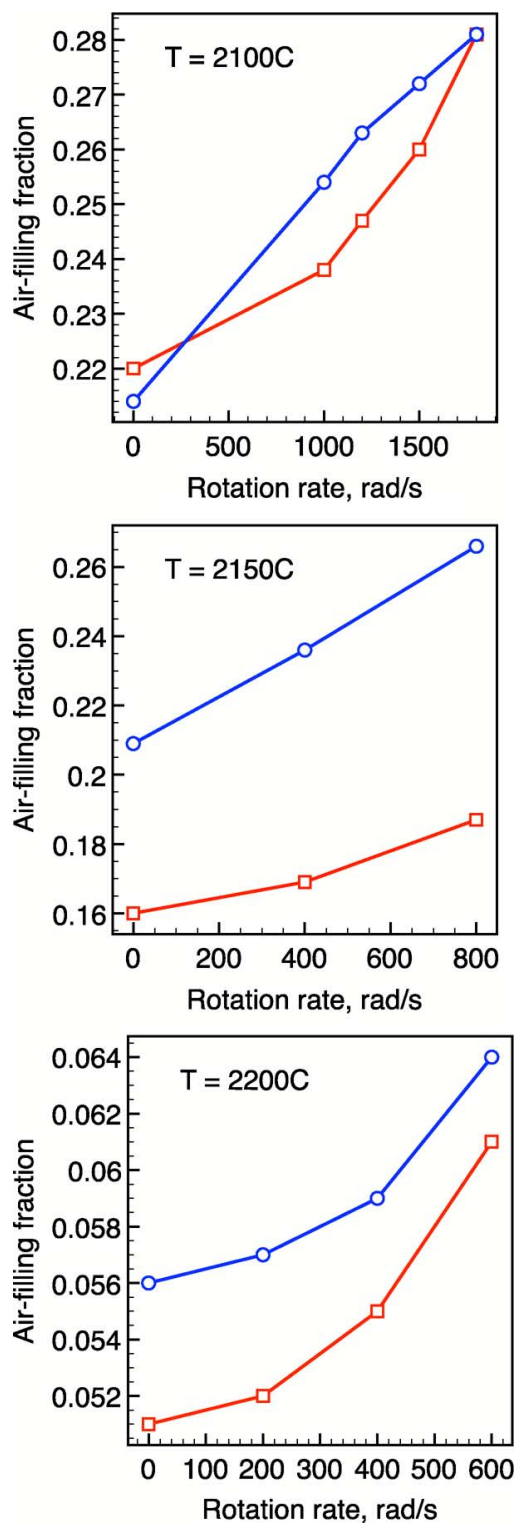

Fig. 5. Graphs showing comparison between predicted (squares) and experimentally observed (circles) air-filling fraction at various capillary tube rotation rates for $\mathrm{T}=2100^{\circ} \mathrm{C}$ (top), $\mathrm{T}=2150{ }^{\circ} \mathrm{C}$ (center) and $\mathrm{T}=2200^{\circ} \mathrm{C}$ (bottom). (Experimental parameters: $h_{10}=1.425 \times 10^{-3}, h_{20}=4.975 \times 10^{-3}, W_{f}=$ $8.83 \times 10^{-5}, W_{d}=1.00 \times 10^{-1}$. Modeling parameters: $h_{10}=1.425 \times 10^{-3}$, $h_{20}=4.975 \times 10^{-3}, W_{f}=8.83 \times 10^{-5}, W_{d}=1.0 \times 10^{-1}, T=2050$, $\gamma=3 \times 10^{-1}, L=3 \times 10^{-2}, g=9.81$. S.I. units are assumed.)

comes increasingly sensitive to rotation rate as the rotation rate increases.

We assume that: (i) the change in inner and outer radii increases with increasing rotation rate; (ii) the change in inner radius is greater than the change in outer radius for any given $B_{0}$ (when compared with the case with no rotation); (iii) the rotation-induced change in inner radius increases with rotation rate at a rate greater than the rate of increase of the change in outer radius; and (iv) $h_{2}>0, h_{1}>0, \Delta h_{2}>0, \Delta h_{1}>0$, and $h_{2}>h_{1}$.

Assumption (i) may be expressed as

$$
\Delta h_{1}=f\left(B_{0}\right)>0, \quad \Delta h_{2}=g\left(B_{0}\right)>0
$$


respectively, where

$$
\frac{\partial f\left(B_{0}\right)}{\partial B_{0}} \geq 0, \quad \frac{\partial g\left(B_{0}\right)}{\partial B_{0}} \geq 0 \text { for } B_{0} \in[0, \infty]
$$

Assumption (ii), $\Delta h_{1}>\Delta h_{2}$, may now be written as

$$
f\left(B_{0}\right)-g\left(B_{0}\right)=k\left(B_{0}\right)>0 \text { for } B_{0} \in[0, \infty] .
$$

Assumption (iii) may be expressed as

$$
\frac{\partial\left(\Delta h_{1}\right)}{\partial B_{0}}>\frac{\partial\left(\Delta h_{2}\right)}{\partial B_{0}}
$$

which, using (6), may be expressed as

$$
\frac{\partial f\left(B_{0}\right)}{\partial B_{0}}-\frac{\partial g\left(B_{0}\right)}{\partial B_{0}}=m\left(B_{0}\right)
$$

where $m\left(B_{0}\right)>0$ for $B_{0} \in[0, \infty]$.

Assumptions (ii) and (iv) give, adding $h_{2}$ to each side and rearranging

$$
\frac{h_{1}+\Delta h_{1}}{h_{2}+\Delta h_{2}}>\frac{h_{1}}{h_{2}}
$$

The left-hand side of (11) is the air-filling fraction with rotation present and the right-hand side is the air-filling fraction without rotation, thus proving (a).

In order to prove (b), we set

$$
Q=\frac{h_{1}+\Delta h_{1}}{h_{2}+\Delta h_{2}}-\frac{h_{1}}{h_{2}}
$$

and are required to show that

$$
\frac{\partial Q\left(B_{0}\right)}{\partial B_{0}}>0 \text { for } B_{0} \in[0, \infty] .
$$

We have that

$$
\frac{\partial Q\left(B_{0}\right)}{\partial B_{0}}=\frac{1}{\left(h_{2}+g\left(B_{0}\right)\right)}\left[\frac{\partial f\left(B_{0}\right)}{\partial B_{0}}-\frac{\partial g\left(B_{0}\right)}{\partial B_{0}} \frac{\left(h_{1}+f\left(B_{0}\right)\right)}{\left(h_{2}+g\left(B_{0}\right)\right)}\right]
$$

which, using assumption (iv) and (10) gives

$$
\frac{\partial Q\left(B_{0}\right)}{\partial B_{0}}>0 \text { for } B_{0} \in[0, \infty]
$$

as required by (13) and thus proving (b), that the air-filling fraction increases monotonically with increasing rotation rates. It is therefore clear that for a given choice of drawing parameters, the amount by which the air-filling fraction increases is related to the amount by which the preform is rotated, though through (b) we establish that the relationship is nonlinear.

\section{B. Extension to MOFs}

The significance of the air-filling fraction to the optical properties of MOFs means that it is particularly important to be able to quantify the effects of rotation on the air-filling fraction of MOF preforms with accuracy. It was shown in [10] that for both large mode area fibers with an approximately uniform distribution of diffuse holes (useful for transmission, for example) and for fibers where a large "jacket" surrounds a microstructured region with a high density of holes, the mathematical model developed herein for capillary tubes may be used to quantify the effects of preform rotation on final geometry. Thus, the good agreement between the model predictions and the experimental results gives confidence in the ability to significantly reduce or remove PMD and fiber birefringence in particular classes of MOF. Furthermore, since numerical simulations accurately predict how preform rotation alters the air-filling fraction, the results of [10] show that the air-filling fraction in certain classes of MOF may be carefully controlled/predicted with preform rotation.

Regarding the extension of these methodologies to general MOFs, there are four principal possibilities, two "coarse grained" and two "fine grained." First, we may draw general conclusions by using the "Type One"/"Type Two" classifications, as put forward in [10]. Second, we may propose a "multiphase flow" model where the averaged properties of the holes are computed and coupled to the flow equations of the host fluid. Third, we may solve an iterative sequence of biharmonic problems (see [14] for details) by slicing the geometry into cross-sections, solving for each cross-section, and coupling the solutions. Finally, it may be possible to solve the general flow equations with a MOF geometry in the asymptotic limit (where the width of a preform is still much less than its length), and directly compute the numerical solution. However, we expect this to be exceedingly computationally intense for even as few as 10 holes. We propose to explore the other three options in further studies.

\section{CONCLUSION}

A mathematical model that includes the effects of inertia, viscosity, surface tension and gravity on the drawing of rotating capillary preforms has been described and solved numerically. The temperature profile of a typical drawing furnace was measured and the viscosity of high-purity silica glass carefully chosen from the published data. This information was used to predict the results of experimental trials, demonstrating a remarkable agreement between the two sets of data. Of particular interest to experimentalists is the air-filling fraction: the effects of preform rotation on the air-filling fraction were described and explained, allowing the air-filling fraction of certain classes of MOF to be predicted and/or controlled by the use of preform rotation. The results of this study clearly demonstrate that a combination of mathematical modeling and careful experimental evaluation of the physical parameters present in the model can provide experimenters with an accurate predictive tool, surpassing the previous engineering "rules of thumb" for parameter windows in fiber manufacture. Furthermore, the excellent agreement between modeling with no fitting parameters used and experiments, verifies the asymptotic modeling techniques used. 


\section{ACKNOWLEDGMENT}

The authors would like to thank Dr. W. Belardi for conducting the experiments described in this paper, and Prof. D. J. Richardson for useful discussions.

\section{REFERENCES}

[1] J. K. Ranka, R. S. Windeler, and A. J. Stentz, "Optical properties of high-delta air-silica microstructure optical fibers," Opt. Lett., vol. 25, no. 11 , pp. 796-798, 2000 .

[2] T. M. Monro, D. J. Richardson, N. G. R. Broderick, and P. J. Bennett, "Holey optical fibers: An efficient modal model," J. Lightw. Technol., vol. 17, no. 6, pp. 1093-1102, Jun. 1999.

[3] T. M. Monro, D. J. Richardson, and P. J. Bennett, "Developing holey fibers for evanescent field devices," Electron Lett., vol. 35, no. 14, pp. $1188-1189,1999$.

[4] A. J. Barlow, J. J. Ramskov-Hansen, and D. N. Payne, "Birefringence and polarization mode-dispersion in spun single-mode fibers," Appl. Opt., vol. 20, no. 17, pp. 2962-2968, 1981.

[5] M. J. Li and D. A. Nolan, "Fiber spin-profile designs for producing fibers with low polarization mode dispersion," Opt. Lett., vol. 23, no. 21, pp. 1659-1661, 1998

[6] R. E. Schuh, X. K. Shan, and A. S. Siddiqui, "Polarization mode dispersion in spun fibers with different linear birefringence and spinning parameters," J. Lightw. Technol., vol. 16, no. 9, pp. 1583-1588, Sep. 1998.

[7] M. Fuochi, J. R. Hayes, K. Furusawa, W. Belardi, J. C. Baggett, T. M. Monro, and D. J. Richardson, "Polarization mode dispersion reduction in spun large mode area silica holey fibres," Opt. Express, vol. 12, no. 9, pp. 1972-1977, 2004.

[8] J. R. Hayes, Personal Communication Univ. Southampton, Optoelectronics Research Centre, Southampton, U.K., 2003.

[9] C. J. Voyce, A. D. Fitt, and T. M. Monro, "The mathematical modelling of rotating capillary tubes for holey-fibre manufacture," J. Eng. Math., vol. 60, no. 1, pp. 68-87, Jan. 2008.

[10] C. J. Voyce, A. D. Fitt, and T. M. Monro, "Mathematical model of the spinning of microstructured fibres," Opt. Express, vol. 12, no. 23, pp. 5810-5820, 2004.

[11] A. D. Fitt, K. Furusawa, T. M. Monro, and C. P. Please, "Modeling the fabrication of hollow fibers: Capillary drawing," J. Lightw. Technol., vol. 19, no. 12, pp. 1924-31, Dec. 2001.

[12] A. D. Fitt, K. Furusawa, T. M. Monro, C. P. Please, and D. J. Richardson, "The mathematical modelling of capillary drawing for holey fibre manufacture," J. Eng. Math., vol. 43, no. 2-4, pp. 201-227, 2002.

[13] C. J. Voyce, A. D. Fitt, and T. M. Monro, "Mathematical modelling of the drawing of spun capillary tubes," in Progress in Industrial Mathematics at ECMI 2002, A. Buikis, R. Ciegis, and A. D. Fitt, Eds. Berlin, Germany: Springer-Verlag, 2004, pp. 387-391.

[14] C. J. Voyce, "The mathematical modelling of microstructured optical fibres," Ph.D. dissertation, Sch. Math., Univ. Southampton, Southampton, U.K., 2005.

[15] R. H. Doremus, "Viscosity of silica," J. Appl. Phys., vol. 92, no. 12, pp. 7619-7629, 2002.
[16] G. Hetherington, K. H. Jack, and J. C. Kennedy, "The viscosity of vitreous silica," Phys. Chem. Glasses, vol. 5, pp. 130-136, 1964.

[17] G. Urbain, Y. Bottinga, and P. Richet, "Viscosity of liquid silica, silicates and alumino-silicates," Geochimica Cosmochimica Acta, vol. 46, no. 6, pp. 1061-1072, 1982.

[18] Module Documentation for the Numerical Algorithms Group (Nag), Limited, Routine d02haf, [Online]. Available: http://gams.nist.gov/ serve.cgi/Module/NAG/D02HAF/681/

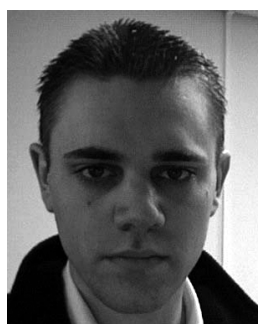

Christopher J. Voyce received the Ph.D. degree from the University of Southampton, Southampton, U.K.

He is currently with the Mathematical Institute, University of Oxford, Oxford, U.K. He was with the University of Adelaide, Adelaide, Australia. His research interests include industrial applied mathematics.

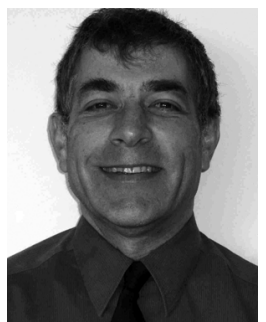

Alistair D. Fitt received the D.Phil. degree from the University of Oxford, Oxford, U.K., in 1983, for his work on the film cooling of turbine blades.

$\mathrm{He}$ is currently Head of the School of Mathematics, University of Southampton, Southampton, U.K. Most of his mathematical career has been spent considering the mathematical modeling of industrial processes. He has also been a frequent contributor to Study Groups with Industry in many different worldwide locations. His other mathematical interests include the study of fluid and solid mechanics in human eyes, and various aspects of betting and gaming.

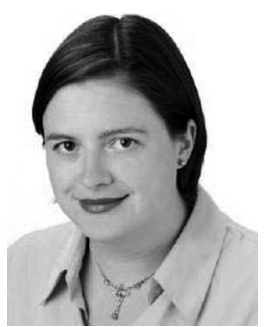

Tanya M. Monro received the Ph.D. degree from the University of Sydney, Sydney, Australia, with a dissertation on self-written waveguides in photosensitive glasses.

Since 2005, she has been the Chair of Photonics and the Director of the Centre of Expertise in Photonics with the School of Chemistry and Physics, University of Adelaide, Adelaide, Australia. From 1998 to 2004, she was with the ORC, the University of Southampton, Southampton, U.K., where she worked on silica and soft glass MOFs. Her current research within the Centre of Expertise in Photonics focuses on the design, fabrication and device applications of new classes of soft glass MOFs, including work on new transmission fibers, highly nonlinear fibers, chem/bio sensing with new fibers and novel fiber lasers. 